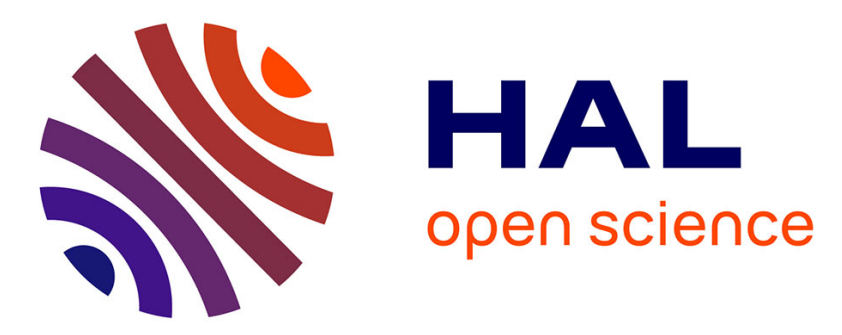

\title{
High quality optical pulse train generator based on solitons on finite background
}

Julien Fatome, Bertrand Kibler, Christophe Finot

\section{To cite this version:}

Julien Fatome, Bertrand Kibler, Christophe Finot. High quality optical pulse train generator based on solitons on finite background. Optics Letters, 2013, 38 (10), pp.1663-1665. 10.1364/OL.38.001663 . hal-00809841

\section{HAL Id: hal-00809841 https://hal.science/hal-00809841}

Submitted on 9 Apr 2013

HAL is a multi-disciplinary open access archive for the deposit and dissemination of scientific research documents, whether they are published or not. The documents may come from teaching and research institutions in France or abroad, or from public or private research centers.
L'archive ouverte pluridisciplinaire HAL, est destinée au dépôt et à la diffusion de documents scientifiques de niveau recherche, publiés ou non, émanant des établissements d'enseignement et de recherche français ou étrangers, des laboratoires publics ou privés. 


\title{
High quality optical pulse train generator based on solitons on finite background
}

\author{
J. Fatome, B. Kibler and C. Finot* \\ Laboratoire Interdisciplinaire CARNOT de Bourgogne, UMR 6303, CNRS-Université de Bourgogne, \\ 9 av. A. Savary, 21078 Dijon, France \\ *Corresponding author: christophe.finot@u-bourgogne.fr
}

Received Month X, XXXX; revised Month X, XXXX; accepted Month X, XXXX; posted Month X, XXXX (Doc. ID XXXXX); published Month X, XXXX

\begin{abstract}
We report a simple method to exploit the typical properties of solitons on finite background in order to generate high repetition rate and high quality optical pulse trains. We take advantage of the nonlinear evolution of a modulated continuous wave towards localized structures upon a nonzero background wave in anomalous dispersive fiber. After a stage of nonlinear compression, a delay-line interferometer enables the annihilation of the finite background and simultaneously allows the repetition rate doubling of the pulse train.
\end{abstract}

OCIS Codes: $190.4370,190.5530,320.5540$

Solitons on finite background (SFB) are solutions of the nonlinear Schrödinger equation (NLSE) that are currently stimulating a renewed and widespread attention. Even if their analytical expressions have been proposed for more than 25 years [1, 2], these nonlinear structures have been only recently identified as promising prototypes of the infamous oceanic rogue waves. Indeed, due to the periodic exchange of energy between a continuous background and a localized structure, an initially small perturbation can exponentially grow into a brief and intense spike before disappearing. With the emergence of the field of optical rogue waves, theoretical and experimental works have confirmed that optical fibers represent an ideal testbed to easily generate and study SFB : Peregrine soliton [3], Akhmediev breathers (ABs) [4], or Kuznetsov-Ma (KM) solitons [5] have indeed been demonstrated taking advantage of widely available components for ultrafast optics.

In this context, it sounds appealing to benefit from the strong temporal compression and peak power increase undergone by an initial perturbation to generate ultrashort pulses and to answer the needs of highly demanding applications such as optical sampling or ultrahigh capacity transmissions. Indeed, pedestal and background-free picosecond pulse trains at repetition rates of several tens of $\mathrm{GHz}$ and with a low duty cycle are required. In this context, using the Kerr nonlinearity acting into an optical fiber has already been demonstrated to be a practical and cost-effective alternative to conventional onerous active mode-locking lasers and several schemes have been demonstrated at various repetition rates $[6,7]$. But to date, except the strategies numerically discussed in [8] by C. Mahnke and F. Mitschke, no solution has been designed to take advantage of the specific features of the SFB and to eliminate the non-negligible background.

Here we propose to fully exploit the nonlinear evolution undergone by a sinusoidal modulation with a finite background propagating along an optical fiber with anomalous dispersion. It has been theoretically shown than such an initial condition reshapes into ultrashort structures close to $\mathrm{AB}$ or $\mathrm{KM}$ solitons depending on the balance between dispersive and nonlinear effects but also on the amplitude of the initial modulation [3, 5]. Our purpose being to generate high repetition pulse trains, it seems more natural to concentrate on the processing of $\mathrm{AB}$ that intrinsically exhibits a temporal periodicity. Note however that the present method could be also applied to a periodic train of KM solitons such as described in [5]. At the point of maximum compression, the initial beating at a frequency $\omega_{\text {mod }}$ is reshaped into the following field [1, 2]:

$$
\psi(t)=\sqrt{P_{0}} \frac{(1-4 a)+\sqrt{2 a} \cos \left(\omega_{\text {mod }} t\right)}{\sqrt{2 a} \cos \left(\omega_{\text {mod }} t\right)-1}
$$

where $P_{0}$ denotes the average power of the initial signal and $a$ is a normalized parameter given by $2 a=1-\left(\omega_{\bmod } / \omega_{c}\right)^{2}$ with $\omega^{2}=4 \gamma P_{0} /\left|\beta_{2}\right|, \beta_{2}$ and $\gamma$ being the dispersive and nonlinear coefficients of the fiber, respectively.

An illustration of the pulse train obtained at the point of maximum compression for $a=0.41$ is given in the panels (a) of Fig. 1. The intensity profile clearly exhibits the ultrashort periodic structures with a low duty cycle (Fig. 1a1). A peak-power being 12 times higher than the continuous background is achieved. The corresponding amplitude $\psi(T)$ (Fig. 1a2) outlines that the background has an opposite sign compared with the modulated part. The optical spectrum presents the triangular shape typical of $\mathrm{AB}$ [4] (Fig. 1a3).

Our original method to cancel the deleterious background is to exploit the $\pi$ phase shift that exists between the pulsed part and the background. Compared to [8], our method is here purely linear. We use here an interferometer not only to double the repetition rate [9] but also to annihilate the deleterious background by 
imprinting a controlled $\pi$ phase shift. Panels (2) illustrate this process and show how the interferential operation appears as destructive for the deleterious background and constructive for the pulsed part. Indeed, after a first splitting stage into both identical replicas, the resulting pulse trains are delayed by half of the period as well as phase shifted by $\pi$. Finally, after recombination, the final pulse train $\psi_{T}(t)$ is given by the following expression:

$$
\psi_{T}(t)=\sqrt{P_{0}} \frac{4(1-2 a) \sqrt{a} \cos \left(\omega_{\bmod } t\right)}{1-2 a \cos ^{2}\left(\omega_{\bmod } t\right)}
$$

and the temporal intensity profile reads as:

$$
P(T)=P_{0} \frac{16 a(1-2 a)^{2} \cos ^{2}\left(\omega_{\bmod } t\right)}{1+4 a^{2} \cos ^{4}\left(\omega_{\bmod } t\right)-4 a \cos ^{2}\left(\omega_{\text {mod }} t\right)}
$$

From Eq. (3), one can make out that between two successive spikes of light, the intensity profile falls back to zero, confirming the total annihilation of the initially continuous background. The intensity profile is close from a hyperbolic secant profile (see magnification of Fig. 1c1), but as proven by Eq. (3) and the deviations observed in the wings of the pulsed part, the resulting field is not here an exact solution anymore of the NLSE. Our method therefore differs from the strategies discussed in [8] to convert $\mathrm{AB}$ into pedestal free solitons. Regarding the peak power of the pulses, it obeys the simple equation: 16 a $P_{0}$.
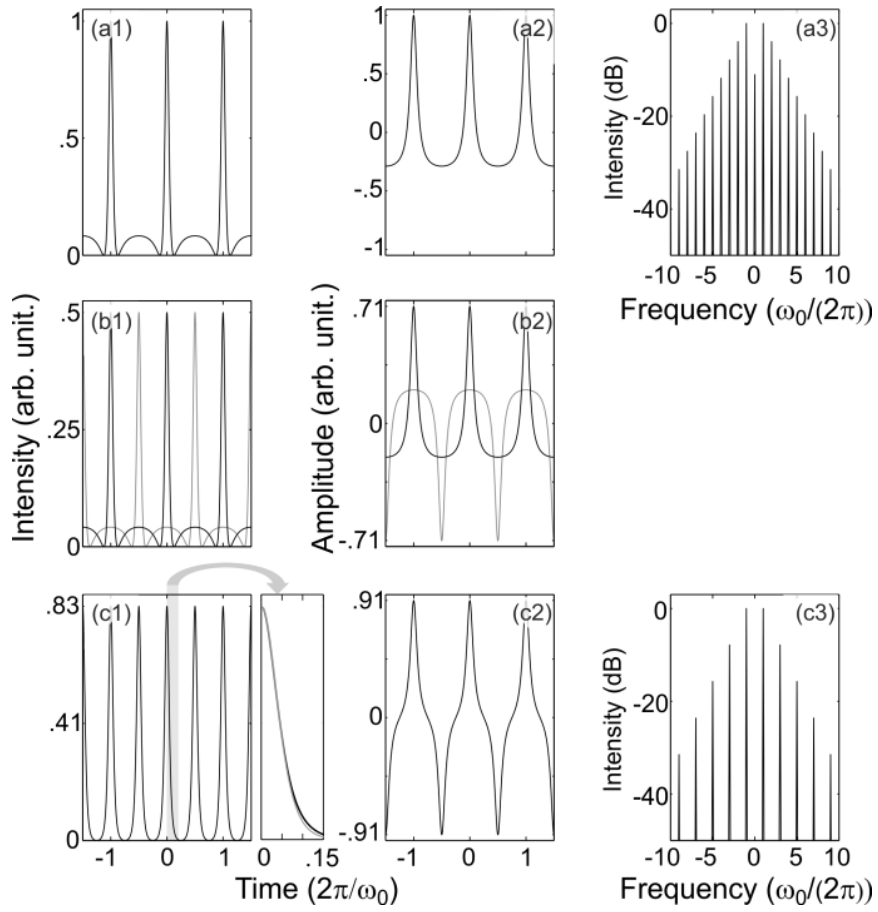

Fig. 1. Principle of frequency doubling and clean up operations on a pulse train on a finite background. (a) Initial train of breathers. (b) Initial train of breathers (black line) and replica delayed by half of a period and phase-shifted by $\pi$ (grey line). (c) Resulting pulse train after recombination. Temporal intensity and amplitude profiles as well as spectral intensity profiles are compared in panels 1, 2 and 3 respectively. In the magnification of (c1), the resulting intensity profile (black line) is compared with the temporal sech intensity profile of a soliton (grey line)
In the spectral domain, as illustrated in panel (c3) of Fig. 1, our linear processing is equivalent to cancel one spectral component over two, which is a clear signature of the doubling of the repetition rate. Note that our pulse train is therefore carrier suppressed, as confirmed by panel (c2) : amplitude of the pulses have alternate signs.

In order to validate this concept, we have experimentally implemented the setup described in Fig. 2 which is based on cost-effective components that are widely used for optical communications. A continuous wave (CW) emitted by an external cavity laser is phase modulated (phase modulator PM) to avoid Brillouin backscattering. The $\mathrm{CW}$ signal is then sinusoidally modulated by means of an intensity modulator (IM) driven by a $25-\mathrm{GHz} \mathrm{RF}$ beating. The amplitude of modulation is voluntary detuned so as to keep a non-total extinction contrast (Fig. 3(a), dotted black line). An erbium-doped fiber amplifier (EDFA) is then used in order to increase the average power up to $28 \mathrm{dBm}$. The reshaping of the sinusoidal beating into SFB is achieved during the propagation in a $2.1 \mathrm{~km}$ standard single mode fiber (SMF-28) with an anomalous dispersion of $-2110^{-3}$ $\mathrm{ps}^{2} / \mathrm{m}$ and a nonlinearity of $1.1 / \mathrm{W} / \mathrm{km}$.

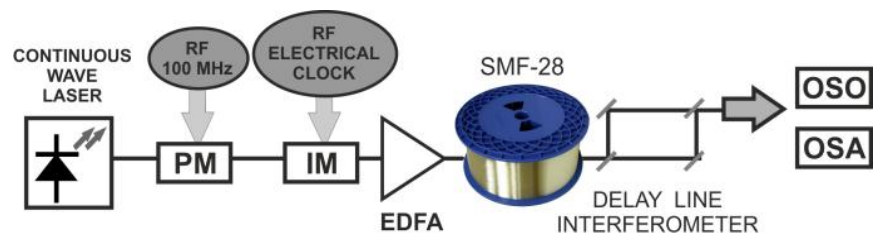

Fig. 2. Experimental setup.

Both temporal and spectral intensity profiles of the resulting pulse train at the maximum of compression are characterized thanks to an optical sampling oscilloscope (OSO) with a picosecond resolution and an optical spectrum analyzer (OSA), respectively. Results are plotted in Fig. 3(a) with dotted grey lines and illustrate the nonlinear reshaping into picosecond pulses characterized by a significant part of energy (25\%) contained in a finite background, thus forbidding any practical application. The full-width at half maximum (FWHM) duration is $3.2 \mathrm{ps}$ indicating a 1/12.5 duty cycle. These experimental observations (Fig. 3a2) are in close agreement with the analytical results predicted from fiber and initial signal properties with $a=0.41$ (panel a1). The optical spectrum exhibits the typical triangular shape expected for SFB.

The delay-line interferometer (DLI) relies on a mechanical delay line that provides a delay which is half the period of the pulse train. A piezo-electrical device incorporated in the interferometer imprints the requested $\pi$ phase-shift. Results of the linear shaping are detailed in Figs. 3 by means of solid black lines and exhibit the efficient doubling of the repetition rate combined with a significant enhancement of the pulse quality. Indeed, the level of the continuous background does not exceed $1 \%$ of 
the peak power and no noticeable pulse broadening occurs. The resulting duty cycle is therefore $1 / 6$, which is comparable with the duty cycle usually obtained by means of other Kerr nonlinearity based techniques starting from an initial beating at $2 \omega_{\text {mod }}[6,7]$. In the spectral domain, the central component is cancelled and the even harmonics disappear with an extinction ratio of $25 \mathrm{~dB}$ corresponding to the interferometer properties.
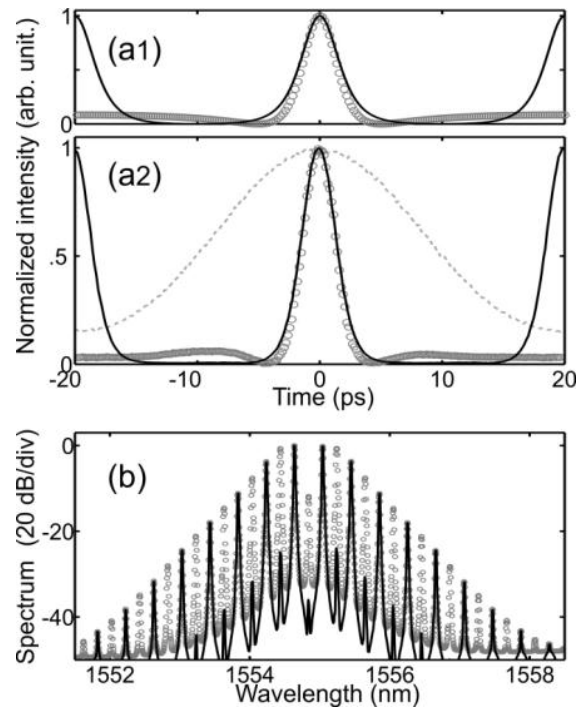

Fig. 3. Experimental results obtained for an input frequency of $25 \mathrm{GHz}$. Evolution of the temporal intensity profile over one period of the initial beat-signal (plotted with grey dashed line) : the pulse train emerging from the SMF (grey circles) is compared with the pulse train obtained after the DLI (solid black line). Analytical predictions from Eq. (1-3) (a1) are compared with the experimental results (a2). (b) Corresponding experimental spectra.

A significant benefit of our approach is the large flexibility of the device regarding its main characteristics, especially the range of repetition rates that can be processed with a fixed segment of fiber. Indeed, contrary to usual methods where the length of the fiber has to be carefully optimized to reach acceptable results, here the same fiber can be used to generate $28-\mathrm{GHz}$ pulse trains (Fig. 4a) up to 80 $\mathrm{GHz}$ pulse trains (Fig. 4b) starting from $14^{-} \mathrm{GHz}$ and $40^{-}$ $\mathrm{GHz}$ electrical clocks, respectively. The level of input powers required to achieve such reshaping varies from 28 up to $30 \mathrm{dBm}$ for duty cycles between 1/6 and 1/3.

Other complementary experiments have been carried out with a longer SMF (length of $8 \mathrm{~km}$ ), in order to achieve lower repetition rates at lower average powers. A selection of the results has been summarized in Figs. 4(cd) and confirms that the principle of the method can be scaled to various repetition rates according to the properties of the fiber involved in the compression stage.

In summary, we have proposed and experimentally demonstrated an easy to implement linear shaping that enables to take full benefits of the properties of solitons on finite background. Taking advantage of the phase shift existing between the continuous background and the pulsed part of the SFB, the present interferential method allows to efficiently remove the background in such a way to generate high quality pulse trains cadenced at a twofold repetition rate. Compared to other techniques based on the usual nonlinear reshaping of an initial beatsignal into an optical fiber which are generally restricted to a particular set of parameters, the combination of SFB generation followed by a interferential post-processing offers a large range of tunability in repetition rate and pulse width for a fixed segment of fiber, while keeping a high level of pulse quality.

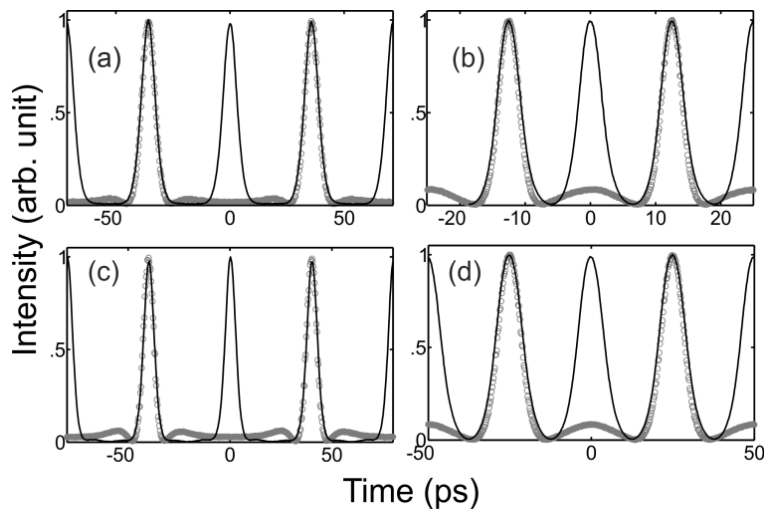

Fig. 4. Experimental results obtained for various repetition rates and fiber lengths. The temporal intensity profile over two periods of the pulse train emerging from the SMF (grey circles) is compared with the pulse train obtained after the DLI (solid black line). Results obtained by means of a $2.1-\mathrm{km}$ long SMF for an output repetition rate of $28 \mathrm{GHz}$ (a) and $80 \mathrm{GHz}$ (b) are compared with results obtained in $8 \mathrm{~km}$ for output frequency of $25 \mathrm{GHz}$ (c) and $40 \mathrm{GHz}(\mathrm{d})$.

We thank K. Hammani and G. Millot for stimulating discussions. We acknowledge the financial support of the Conseil Regional de Bourgogne (Pari Photcom) and the Agence Nationale de la Recherche (project SO FAST, ANR-11-EMMA-0005). We also thank the technical support of Photline Technologies for providing the interferometer.

\section{References}

1. E. A. Kuznetsov, Doklady Akamemii Nauk SSSR, 1977. 22: p. 507-508.

2. N. N. Akhmediev and V.I. Korneev, Theor. Math. Phys., 1986. 69(2): p. 1089-1093.

3. K. Hammani, B. Kibler, C. Finot, P. Morin, J. Fatome, J. M. Dudley, and G. Millot, Opt. Lett., 2011. 36(2): p. 112114.

4. K. Hammani, B. Wetzel, B. Kibler, J. Fatome, C. Finot, G. Millot, N. Akhmediev, and J. M. Dudley, Opt. Lett., 2011. 36(11): p. 2140-2142.

5. B. Kibler, J. Fatome, C. Finot, G. Millot, G. Genty, B. Wetzel, N. Akhmediev, F. Dias, and J. M. Dudley, Sci. Rep., 2012. 2: p. 463.

6. T. Inoue and S. Namiki, Laser \& Photonics Rev., 2008. 2(1): p. 83-99.

7. S. Pitois, C. Finot, J. Fatome, and G. Millot, Opt. Commun., 2006. 260(1): p. 301-306.

8. C. Mahnke and F. Mitschke, Physical Review A, 2012. 85(3): p. 033808.

9. D. K. Serkland, G. D. Bartolini, W. L. Kath, P. Kumar, and A. V. Sahakian, "Rate multiplication of a $59-\mathrm{GHz}$ soliton source at 1550 nm," J. Lightw. Technol. 16, 670-677 (1998). 